\title{
An Unsupervised Approach Based on the Generalized Gaussian Model to Automatic Change Detection in Multitemporal SAR Images
}

\author{
Yakoub Bazi, Student Member, IEEE, Lorenzo Bruzzone, Senior Member, IEEE, and Farid Melgani, Member, IEEE
}

\begin{abstract}
In this paper, we present a novel automatic and unsupervised change-detection approach specifically oriented to the analysis of multitemporal single-channel single-polarization synthetic aperture radar (SAR) images. This approach is based on a closed-loop process made up of three main steps: 1) a novel preprocessing based on a controlled adaptive iterative filtering; 2) a comparison between multitemporal images carried out according to a standard log-ratio operator; and 3) a novel approach to the automatic analysis of the log-ratio image for generating the change-detection map. The first step aims at reducing the speckle noise in a controlled way in order to maximize the discrimination capability between changed and unchanged classes. In the second step, the two filtered multitemporal images are compared to generate a log-ratio image that contains explicit information on changed areas. The third step produces the change-detection map according to a thresholding procedure based on a reformulation of the Kittler-Illingworth (KI) threshold selection criterion. In particular, the modified KI criterion is derived under the generalized Gaussian assumption for modeling the distributions of changed and unchanged classes. This parametric model was chosen because it is capable of better fitting the conditional densities of classes in the log-ratio image. In order to control the filtering step and, accordingly, the effects of the filtering process on change-detection accuracy, we propose to identify automatically the optimal number of despeckling filter iterations [Step 1)] by analyzing the behavior of the modified KI criterion. This results in a completely automatic and self-consistent change-detection approach that avoids the use of empirical methods for the selection of the best number of filtering iterations. Experiments carried out on two sets of multitemporal images (characterized by different levels of speckle noise) acquired by the European Remote Sensing 2 satellite SAR sensor confirm the effectiveness of the proposed unsupervised approach, which results in change-detection accuracies very similar to those that can be achieved by a manual supervised thresholding.
\end{abstract}

Index Terms-Change detection, generalized Gaussian (GG) distribution, multitemporal synthetic aperture radar (SAR) images, threshold selection.

\section{INTRODUCTION}

$\mathbf{T}$ HE DETECTION of changes occurring on the earth surface through the use of multitemporal remote sensing images is one of the most important applications of remote sensing technology. This depends on the fact that, for many public and

Manuscript received May 25, 2004; revised December 15, 2004. This work was supported by the Italian Ministry of Education, University and Research (MIUR).

The authors are with the Department of Information and Communication Technology, University of Trento, I-38050 Trento, Italy (e-mail: lorenzo. bruzzone@ing.unitn.it; melgani@dit.unitn.it).

Digital Object Identifier 10.1109/TGRS.2004.842441 private institutions, the knowledge of the dynamics of either natural resources or man-made structures is a valuable source of information in decision making. In this context, satellite and airborne remote sensing sensors have proved particularly useful in addressing change-detection applications related to environmental monitoring [1], agricultural surveys [2], urban studies [3], and forest monitoring [4].

Usually, change detection involves the analysis of two coregistered remote sensing images acquired over the same geographical area at different times. Such an analysis is called unsupervised when it aims at discriminating between two opposite classes (which represent changed and unchanged areas) without any prior knowledge about the scene (i.e., no ground truth is available for modeling the classes). In the analysis of multitemporal remote sensing data acquired by (optical) multispectral sensors, various automatic and unsupervised change-detection methods have been developed and described in the literature. Most are based on the so-called "difference image" (DI). The most popular way of generating the DI is by change vector analysis (CVA) [5]. This technique exploits a simple vector subtraction operator to compare pixel-by-pixel the two multispectral images under analysis. In some cases, depending on the specific type of changes to be identified, the comparison is made on a subset of the spectral channels. The separation between changed and unchanged classes is done on the magnitude of the resulting spectral change vectors by means of empirical or theoretical well-founded thresholding strategies [5]. In [6], two automatic techniques based on the Bayes theory for the analysis of the DI are proposed. One allows an automatic selection of the decision threshold maximizing the overall change-detection error under the assumption that pixels in the DI are spatially independent. In the hypothesis of Gaussian distribution for changed and unchanged classes, the estimation of the parameters of the Gaussian model is carried out using the expectation-maximization (EM) algorithm [7]. The other technique performs the analysis of the DI using a Markov random field (MRF) approach that exploits the interpixel class dependency in the spatial domain to improve the accuracy of the final change-detection map according to the use of a regularization term. This approach has been extended in [8] by adopting a semiparametric technique that exploits the reduced Parzen estimation (RPE) technique for a better modeling of the density functions associated with changed and unchanged pixels. In [9], the observed multitemporal images are modeled as MRFs in order to search for an optimal image of changes by means of the maximum a posteriori probability (MAP) decision criterion and the simulated annealing (SA) energy minimization procedure. 
In [10], a comparative study is presented on the effectiveness of different thresholding algorithms developed for general pattern recognition problems (i.e., Kittler and Illingworth's, Huang and Wang's, and Otsu's algorithms) when applied to change detection in multispectral remote sensing data.

Synthetic aperture radars have been less exploited than optical sensors in the context of change detection. This is due to the fact that SAR images suffers from the presence of the speckle noise that makes it difficult to analyze such imagery, and in particular to perform unsupervised discrimination between changed and unchanged classes. Despite the presence of speckle noise, the use of SAR sensors in change detection is potentially attractive from the operational viewpoint. These active microwave sensors present the advantage that (unlike optical ones) they are independent of atmospheric and sunlight conditions. This means that they are capable of monitoring geographical areas regularly (even if covered by clouds) and also of controlling polar regions even during the local winter period when solar light is severely limited. This makes it possible to plan the monitoring of a region (by repeat-pass imaging) with advance timing defined according to end-user requirements (e.g., seasonal and agricultural calendars).

In the context of SAR image analysis, the problem of unsupervised change detection has been addressed focusing on different aspects, including image despeckling, choice of the comparison operator, and optimal threshold selection. In [11], the authors show the usefulness of multitemporal SAR images acquired over an annual cycle to monitor the changes occurring on the land surface. The change-detection task is carried out by a simple thresholding of the cumulative histogram of the difference image on the basis of predefined threshold values. In order to minimize the speckle effect, the authors applied a $5 \times 5$ mean filter to the difference image. Because of the multiplicative nature of speckle noise, it appears more effective to use the ratio operator than the difference operator to compare two SAR temporal images [12], [13]. A method based on the generation of a logarithmic-scaled ratio image from a couple of multitemporal SAR images, followed by an adaptive filtering and a simple thresholding procedure applied according to a "manual" selection of the decision threshold, is presented in [14]. In [15], the original images are preprocessed in different ways (i.e., block averaging, Gamma MAP filter, image segmentation), and changes are identified by "manually" thresholding the log-ratio image generated from the filtered images according to a desired probability of false alarms. In [16], the suitability of the principal component analysis (PCA) method for change detection is investigated. Here the filtering is carried out using the Gamma-Gamma MAP filter. PCA is then applied in two different ways by combining: 1) the original multitemporal despeckled images and 2) a set of three images made up of intensity ratio, amplitude ratio, and backscattering coefficient $\sigma^{0}$ difference images. The authors show that, in the first case, the changed class cannot be isolated by PCA, whereas, in the second case, the first component accounts for most of the temporal change. In [17], change detection is carried out in order to identify forested areas that underwent storm damage. The authors use a linear regression model to estimate the radar backscattering amplitudes from the inventoried stem volumes before the storm. Such estimates were then compared with the radar backscattering amplitudes measured after the storm using a statistical evaluation based on the Student's t-test to discriminate between wind-thrown forests and unaffected forests. Dierking and Skriver [18] found that, in multitemporal polarimetric SAR data, intensity images are better suited for change-detection purposes than correlation coefficient and phase difference between the copolarized channels. In particular, they used the ratio operator to compare the temporal images and a decision mechanism based on a desired value of the probability of false alarms. In [19], four different features (the temporal ratio, second-, and third-order Log-cumulants, and a mixture parameter of two Gamma distributions) are extracted from a series of seven multitemporal SAR images and studied for change-detection purposes. The unsupervised discrimination between changed and unchanged classes was carried out using the K-means clustering algorithm. The experimental results show that the temporal ratio is well suited to detect sharp changes such as flooded areas, while it is not effective to monitor progressive or seasonal changes, for which, on the other hand, the second-order log-cumulant and the mixture level parameters seem useful.

In general, it appears clearly from the literature that the main unsolved problem with change detection in SAR imagery is the lack of accurate and reliable methods capable of performing unsupervised change detection in a completely automatic way. To overcome this problem, in this paper we propose a novel automatic unsupervised change-detection approach specifically oriented to the analysis of single-channel single-polarization SAR images. This approach, which is suitable for the detection of distributed changes, is based on a closed-loop process consisting of three main steps: 1) a novel controlled preprocessing based on adaptive filtering; 2) a comparison between multitemporal images; and 3) a novel technique for the automatic analysis of the image resulting from the comparison. The first step aims at reducing speckle noise in a controlled manner in order to maximize the discrimination capability between changed and unchanged classes. Filtering effects are controlled by exploiting a performance index evaluated in the third step. In the second step, the filtered multitemporal images are compared through the ratio operator to generate a ratio image represented in the logarithmic scale. In the third step, changes are identified by analyzing the log-ratio image according to a novel thresholding procedure. The latter is based on the Kittler-Illingworth (KI) threshold selection criterion originally developed under the Gaussian assumption for the binarization of general-purpose images [20]. This criterion has been adapted to the properties of the considered problem using the generalized Gaussian (GG) assumption for modeling the statistical distributions of changed and unchanged classes. In addition, in order to take into account the effects of the filtering process on change-detection accuracy, we propose to identify the optimal number of filtering iterations (carried out in the first step of the proposed approach) automatically by analyzing the behavior of the modified KI criterion over the filtering iterations. Such a performance index avoids the use of empirical methods for the selection of the number of filtering iterations.

This paper is organized in six sections. Section II introduces the general formulation of the problem and the basics of the proposed approach. Section III describes the procedure developed 


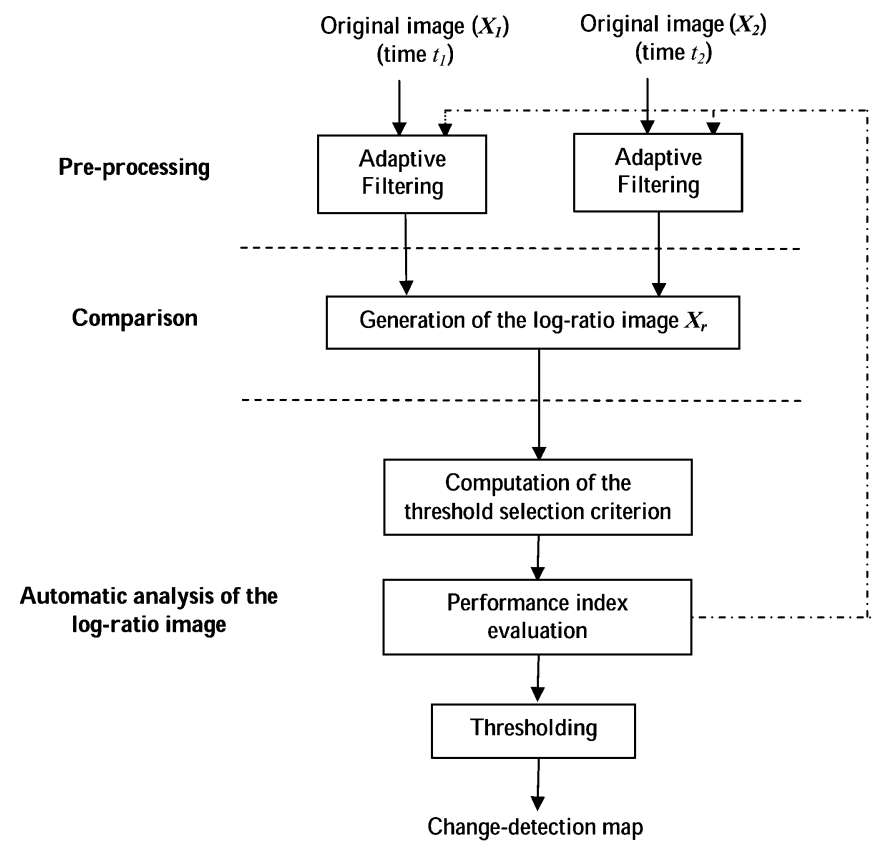

Fig. 1. General block diagram of the proposed change-detection approach.

for automatic selection of the decision threshold in the log-ratio image and for generating the final change-detection map. The datasets used are presented in Section IV, which also contains a description of the experiments. The results obtained for the first and second datasets are reported in Section V. Finally, conclusions are drawn in Section VI.

\section{Problem Formulation ANd General Description OF THE PROPOSED APPROACH}

Let us consider two coregistered SAR intensity (or amplitude) images $X_{1}=\left\{X_{1}(i, j), 1 \leq i \leq I, 1 \leq j \leq J\right\}$ and $X_{2}=\left\{X_{2}(i, j), 1 \leq i \leq I, 1 \leq j \leq J\right\}$ acquired over the same geographical area at two different times $t_{1}$ and $t_{2}$, respectively. We aim at generating a change-detection map that represents changes that occurred on the ground between the acquisition dates of the two images $X_{1}$ and $X_{2}$. The change-detection problem can be modeled as a binary classification problem where each pixel is mapped into the set $\Omega=\left\{\omega_{u}, \omega_{c}\right\}$ of possible labels $\left(\omega_{u}\right.$ and $\omega_{c}$ represent the unchanged and changed classes, respectively). Let us assume that no ground truth is available to estimate the statistical models associated with these two classes. The proposed automatic and unsupervised changedetection approach is made up of three main steps (see Fig. 1): 1) controlled preprocessing based on adaptive filtering; 2 ) comparison of the pair of multitemporal images; and 3) automatic analysis of the log-ratio image. A detailed description of these different steps is provided in Sections II-A-C.

\section{A. Step 1: Controlled Preprocessing Based on Adaptive Filtering}

This first step aims at reducing speckle noise in a controlled manner so as to maximize the discrimination capability between the changed and unchanged classes represented in the SAR images. In general, a multilooking process (which consists of reducing speckle noise during image formation at the expense of spatial resolution) is applied to most of the SAR images. However, a further filtering step is usually required to make the images suitable for the desired analysis. To this purpose, various filters have been proposed in the SAR literature, among which we recall the Lee [21], the Kuan [22], the Frost [23], and the MAP filters [24]. In this paper, we use the adaptive enhanced Lee filter, which has proved effective for speckle reduction [25]. However, the proposed approach is general and can be used with all kinds of adaptive speckle filters. The main problem with SAR image filtering is related to the determination of the best number of filtering iterations, which is often found empirically. In this paper, we propose to control the filtering process by automatically finding the optimal number of filtering iterations for the specific change-detection application considered. The underlying idea consists of exploiting the KI criterion adopted in the threshold selection procedure, as it is directly related to error probability [10], [20]. This criterion is computed versus the filtering iterations and used as an index of performance of the filtered images in terms of change-detection error. The final change-detection map is generated on the basis of the filtered images that provide the smallest performance index value. We refer the reader to Section III for a detailed description of this step.

\section{B. Step 2: Comparison of Multitemporal Images}

When detecting changes in multispectral remote sensing images, the customary way of comparing a pair of multitemporal images is to generate a difference image by applying a pixel-bypixel subtraction [image differencing (ID) technique]. However, when SAR images are considered, changes are obtained by analyzing the image resulting from the application of the ratio operator to the considered couple of temporal SAR images [12]. It is possible to prove that, under the symplifying asumption of statistical independence between the intensity images $X_{1}$ and $X_{2}$, the distribution of the ratio image $X_{r}$ can be written as follows [12]:

$$
p\left(X_{r}\right)=\frac{(2 N-1) !}{(N-1) !^{2}} \frac{\bar{X}_{r}^{N} X_{r}^{N-1}}{\left(\bar{X}_{r}+X_{r}\right)^{2 N}}
$$

where $N$ is the equivalent number of looks (ENL). The ratio operator shows two main advantages over the difference operator. The first is that the ratio image distribution depends only on the relative change $\bar{X}_{r}=m_{2} / m_{1}$ in average intensity between two dates and not on the intensity level of the pixels, in contrast with the distribution of the difference image [12]. This means that changes will be detected in the same manner both in high and low intensity regions. The second advantage is that the ratio operator is more robust to calibration errors than the difference operator. Since radiometric errors that usually occur during the SAR processing phase are of the multiplicative type and are exactly reproduced in repeat-pass imagery, they can be eliminated using the ratio operator. Furthermore, it is worth noting that, in the literature, the ratio image is usually expressed in a logarithmic scale in order to compress the range of variation of the ratio image and to better balance the values below and above one. Based on these considerations, we adopt the log-ratio operator in the proposed change-detection approach. 


\section{Step 3: Automatic Analysis of the Log-Ratio Image}

The purpose of analyzing the log-ratio image is to discriminate between the two opposite classes $\omega_{c}$ and $\omega_{u}$ (associated with changed and unchanged pixels, respectively). This problem can be formulated in the context of the Bayesian decision theory. The latter requires that for each pixel of the log-ratio image $X_{l}$, both the conditional probability density functions ( $\mathrm{pdf}), p\left(X_{l} \mid\right.$ $\left.\omega_{c}\right)$ and $p\left(X_{l} \mid \omega_{u}\right)$, and the prior probabilities $P\left(\omega_{c}\right)$ and $P\left(\omega_{u}\right)$ of the classes $\omega_{c}$ and $\omega_{u}$, respectively, should be estimated. Such statistical quantities can be related to the pdf $p\left(X_{l}\right)$ associated with the log-ratio image using the total probability theorem, i.e., looking at $p\left(X_{l}\right)$ as a mixture of two density functions associated with the classes $\omega_{c}$ and $\omega_{u}$, i.e.,

$$
p\left(X_{l}\right)=p\left(X_{l} \mid \omega_{c}\right) P\left(\omega_{c}\right)+p\left(X_{l} \mid \omega_{u}\right) P\left(\omega_{u}\right) .
$$

A common simplification adopted in the literature to tackle this binary discrimination problem is based on the pixel independence assumption in the spatial domain. Accordingly, this issue can be reduced to a binary thresholding problem, since the two classes are defined over a one-dimensional feature domain. In this context, a general algorithm for threshold selection is the one proposed by $\mathrm{KI}$, which derives the decision threshold according to an implicit parametric estimation of the statistical model of the two classes under the Gaussian distribution assumption [20]. Despite the fact that the KI algorithm is based on biased estimates of the class distributions, its simplicity and effectiveness make it particularly attractive for change-detection applications. Accordingly, in this paper, we propose to adapt this basic thresholding procedure to deal with the problem of automatic threshold selection in the log-ratio SAR image as described in Section III.

\section{Automatic Threshold SElECtion Procedure}

In this section, we describe the modified KI algorithm for the estimation of the decision threshold between changed and unchanged classes. Let $h\left(X_{l}\right)\left(X_{l}=0,1, \ldots, L-1\right)$ be the histogram of the log-ratio SAR image (we assume that $h\left(X_{l}\right)$ is the only available information about the image). $L$ stands for the number of possible gray levels. The histogram $h\left(X_{l}\right)$ can be considered an approximation of the actual probability density function $p\left(X_{l}\right)$ of the mixture population describing the changed and unchanged pixels. In this work, for simplicity, we assume that changes occur only on one side of the histogram. This assumption does not hold for all change-detection applications, but is often realistic for a large number of them (it is worth noting that the proposed approach can be extended to the case of changes occurring on both sides of the histogram). In the $\mathrm{KI}$ algorithm, the selection of an appropriate decision threshold $T \in\{0,1, \ldots, L-1\}$ is based on the optimization of a predefined function $J(T)$ that averages a cost function $c\left(X_{l}, T\right)$ over the histogram $h\left(X_{l}\right)$. The function $c\left(X_{l}, T\right)$ measures the cost of classifying pixels by comparing their gray-levels $X_{l}$ with the threshold $T$. The KI criterion function $J(T)$ is given by

$$
J(T)=\sum_{X_{l}=0}^{L-1} h\left(X_{l}\right) c\left(X_{l}, T\right)
$$

where

$$
c\left(X_{l}, T\right)= \begin{cases}-2 \ln P\left(\omega_{u} \mid X_{l}, T,\right) & \text { if } X_{l} \leq T \\ -2 \ln P\left(\omega_{c} \mid X_{l}, T\right), & \text { if } X_{l}>T\end{cases}
$$

in which $P\left(\omega_{i} \mid X_{l}, T\right)(i=u, c)$ are the posterior probabilities of the unchanged and changed classes, respectively, given the gray level $X_{l}$ and a specific value of the threshold $T$. The optimal threshold that minimizes the classification error is the one that minimizes the following cost function:

$$
T^{*}=\underset{T=0,1, \ldots L-1}{\arg \min } J(T) .
$$

Using the Bayes theorem, the posterior probability can be expressed in terms of the prior probability and the class-conditional pdf, i.e.,

$$
P\left(\omega_{i} \mid X_{l}, T\right)=\frac{P\left(\omega_{i}\right) \cdot p\left(X_{l} \mid \omega_{i}, T\right)}{\sum_{j \in\{u, c\}} P\left(\omega_{j}\right) \cdot p\left(X_{l} \mid \omega_{j}, T\right)}, \quad i=u, c .
$$

Depending on the model adopted to estimate the class-conditional pdfs, different cost functions can be defined. In particular, in this paper, two kinds of distributions are considered, namely the Gaussian and the generalized Gaussian models. It is worth noting that the reformulation of the KI threshold selection algorithm according to the latter distribution model is one of the novel methodological contributions presented in this work.

\section{A. Threshold Selection Based on the Gaussian Model}

The KI threshold selection algorithm requires a parametric model to describe the statistical distributions of both changed and unchanged classes. A simple and popular model that can represent a possible candidate for this task is the Gaussian distribution. Though one can expect a mixture of two Gaussian distributions not to be precise in reconstructing the statistical behavior of the two classes in the log-ratio image, the Gaussian model may reveal a first simple and reasonable approximation. This is explained by the fact that the distribution of the log-ratio image is expected to be close to a Gaussian distribution [14].

Under the assumption that the class-conditional distributions $p\left(X_{l} \mid \omega_{i}, T\right)(i=u, c)$ follows a Gaussian statistical behavior, the cost function to be optimized is the original one derived by $\mathrm{KI}$ and is given by [20]

$$
\begin{aligned}
J(T)=1+2\left[P_{u}(T) \ln \sigma_{u}(T)+P_{c}(T) \ln \sigma_{c}(T)\right] & \\
& +2 H(\Omega, T)
\end{aligned}
$$

In this case, the parameters to be estimated are the prior probabilities $P_{u}(T)$ and $P_{c}(T)$, the means $m_{u}(T)$ and $m_{c}(T)$, and the variances $\sigma_{u}^{2}(T)$ and $\sigma_{c}^{2}(T)$ associated with the unchanged and changed classes, respectively, for a given value of the threshold $T \in[0, L-1] . H(\Omega, T)$ stands for the entropy associated with 
the binary set of classes $\Omega=\left\{\omega_{u}, \omega_{c}\right\}$. These parameters are estimated according to the following system of equations [20]:

$$
\left\{\begin{array}{l}
P_{u}(T)=\sum_{X_{l}=0}^{T} h\left(X_{l}\right), \quad m_{u}(T)=\frac{1}{P_{u}(T)} \sum_{X_{l}=0}^{T} X_{l} h\left(X_{l}\right) \\
\sigma_{u}^{2}(T)=\frac{1}{P_{u}(T)} \sum_{X_{l}=0}^{T}\left[X_{l}-m_{u}(T)\right]^{2} h\left(X_{l}\right) \\
P_{c}(T)=1-P_{u}(T), \quad m_{c}(T)=\frac{1}{P_{c}(T)} \sum_{X_{l}=T+1}^{L-1} X_{l} h\left(X_{l}\right) \\
\sigma_{c}^{2}(T)=\frac{1}{P_{c}(T)} \sum_{X_{l}=T+1}^{L-1}\left[X_{l}-m_{c}(T)\right]^{2} h\left(X_{l}\right) .
\end{array}\right.
$$

\section{B. Threshold Selection Based on the Generalized Gaussian Model}

In order to improve the threshold selection process, a more general parametric model capable of describing as better as possible the statistical behaviors of changed and unchanged classes in the log-ratio image must be used. Although theoretically these distributions are expected to be close to Gaussian functions, in practice the Gaussian approximation does not accurately match the aforementioned conditional densities of classes. For this reason, we considered an alternative model that should satisfy two main properties: 1) flexibility and robustness (i.e., it should be capable of spanning a large variety of statistical behaviors); 2) stability (i.e., it should not require the estimation of an excessively large number of parameters). Among the possible models, the generalized Gaussian (GG) distribution is a particularly attractive candidate, since it can approximate a large class of statistical distributions (e.g., impulsive, Laplacian, Gaussian, uniform distributions), and it requires the estimation of only one more parameter compared to the Gaussian model. The analytical expression of the GG distribution considered in our approach for modeling the two class-conditional pdfs is given by [26], [27]

$$
p\left(X_{l} \mid \omega_{i}\right)=a_{i} e^{-\left[b_{i}\left|X_{l}-m_{i}\right|\right]^{\beta_{i}}}, \quad i=u, c
$$

where the positive constants $a_{i}$ and $b_{i}$ are given by

$$
a_{i}=\frac{b_{i} \beta_{i}}{2 \Gamma\left(\frac{1}{\beta_{i}}\right)} \quad b_{i}=\frac{1}{\sigma_{i}} \sqrt{\frac{\Gamma\left(\frac{3}{\beta_{i}}\right)}{\Gamma\left(\frac{1}{\beta_{i}}\right)}} .
$$

The terms $m_{i}, \sigma_{i}^{2}$ and $\beta_{i}$ are the mean, the variance, and the shape parameters of the distribution, respectively, and $\Gamma(\cdot)$ is the well-known Gamma function (i.e., $\Gamma(z)=\int_{0}^{\infty} e^{-t} t^{z-1} d t$ ). The shape parameter $\beta_{i}\left(\beta_{i} \geq 0\right)$ tunes the decay rate of the density function. It is worth noting that $\beta_{i}=2$ yields the Gaussian density function and $\beta_{i}=1$ corresponds to the Laplacian density function. The two limit cases $\beta_{i} \rightarrow 0$ and $\beta_{i} \rightarrow \infty$ approach an impulsive function and uniform distribution, respectively (Fig. 2). In order to compute the shape parameters $\beta_{i}(i=u, c)$, we use the estimation technique described in [26]. According to this technique, the shape parameter related to the unchanged class is estimated using the following steps (the same procedure is adopted for the changed class).

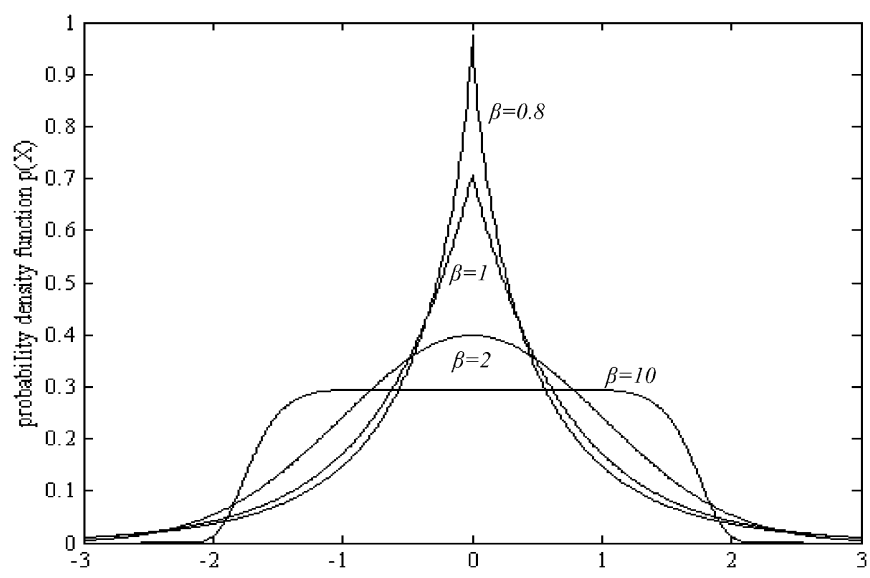

Fig. 2. Expected behavior of the normalized GG distribution $(m=0, \sigma=1)$ versus the value of the shape parameter $\beta$.

Step 1) Generation of a lookup table by computing the so-called generalized Gaussian ratio function $r\left(\beta_{u}\right)$ given by

$$
r\left(\beta_{u}\right)=\frac{\Gamma\left(\frac{1}{\beta_{u}}\right) \Gamma\left(\frac{3}{\beta_{u}}\right)}{\Gamma^{2}\left(\frac{2}{\beta_{u}}\right)} .
$$

Step 2) Determination of the estimate for the modified mean of the absolute values

$$
E\left[\left|X_{l}\right| \mid \omega_{u}\right]=\frac{1}{P_{\left(\omega_{u}\right)}} \sum_{X_{l}=0}^{T} h\left(X_{l}\right) \cdot\left|X_{l}-m_{u}\right| .
$$

Step 3) Computation of the ratio

$$
\rho_{u}=\frac{\sigma_{u}^{2}}{E^{2}\left[\left|X_{l}\right| \mid \omega_{u}\right]} .
$$

Step 4) Identification of the solution to the equation $\hat{\beta}_{u}=$ $r^{-1}\left(\rho_{u}\right)$ using the lookup table.

Following the same reasoning as for the Gaussian distribution, it can be proved that the cost function to be optimized under the GG distribution assumption is as follows (see Appendix):

$$
\begin{aligned}
J(T)= & \sum_{X_{l}=0}^{T} h\left(X_{l}\right)\left[b_{u}(T)\left|X_{l}-m_{u}(T)\right|\right]^{\beta_{u}(T)} \\
& +\sum_{X_{l}=T+1}^{L-1} h\left(X_{l}\right)\left[b_{c}(T)\left|X_{l}-m_{c}(T)\right|\right]^{\beta_{c}(T)} \\
& +H(\Omega, T)-\left[P_{u}(T) \ln a_{u}(T)+P_{c}(T) \ln a_{c}(T)\right] .
\end{aligned}
$$

\section{Generation of the Final Change-Detection Map}

As stated in Section II, depending on the intensity of the speckle, an appropriate number of filtering iterations should be applied to the original images to obtain an accurate change-detection map. The determination of the optimal number of filtering iterations is a critical issue since: 1 ) it should correspond to a good tradeoff between noise reduction and degradation of the spatial details present in the analyzed images; and 2) it should be done in an unsupervised way. Generally, the number 


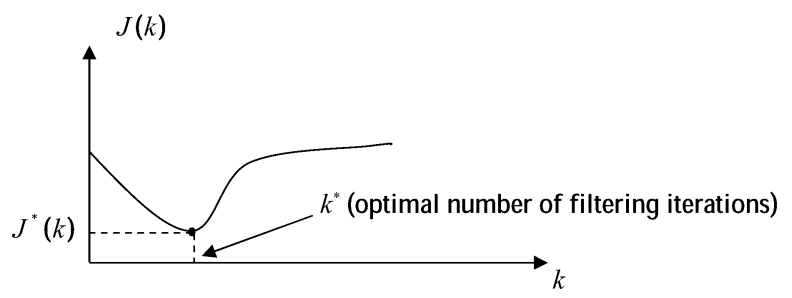

Fig. 3. Behavior of the performance index $J(k)$ versus the number $k$ of filtering iterations.

of iterations is selected in the preprocessing phase either by trial-and-error or according to empirical strategies. As no training pixels are available to determine with precision the best number of filtering iterations, we propose to control the effect of the filtering process on the change-detection results by analyzing the behavior of the cost function $J(T)$. The exploitation of this cost function is motivated by the fact that it is directly related to the change-detection error probability. In particular, we propose to use the minimum value $J(k)$ obtained from the cost function at each filtering iteration $k$ as a performance index to monitor the filtering process, i.e., to find the number of filtering iterations that results in the lowest change-detection error in the proposed technique.

Let $X_{1}^{k}$ and $X_{2}^{k}$ be the two SAR images obtained after filtering $k$ times the original SAR images $X_{1}$ and $X_{2}$, respectively. Let $X_{l}^{k}$ be the log-ratio SAR image generated by applying a proper operator to $X_{1}^{k}$ and $X_{2}^{k}$, and $J(k)$ the minimum value of the cost function obtained for the log-ratio image $X_{l}^{k}$. As shown in Fig. 3, the values of the performance index $J(k)$ are expected to be higher before starting the despeckling process. This is due to statistical overlapping between changed and unchanged classes in the log-ratio image. In fact, the presence of speckle renders the separation between the two classes difficult. We expect that a decrease in the values of $J(k)$ is obtained after the filtering operation, since changed and unchanged classes increase their separability, and consequently they can be modeled more accurately. However, by increasing the number of filtering iterations too much, two undesired effects can be caused: 1) geometrical details can become degraded (small areas of the same class may be completely lost); 2) an overlap can be created between the two classes along their spatial boundaries. This situation leads to an increase in the value of $J(k)$. Consequently, we expect that an appropriate tradeoff between speckle reduction and detail preservation can be obtained on the basis of the minimization of the performance index. This process is carried out in a completely unsupervised way. It is worth noting that the proposed approach can be used with any adaptive speckle filter. The adoption of filters with different effectiveness will result in the identification of different numbers of "optimal" iterations and therefore in a different tradeoff between accuracy in homogeneous areas and the preservation of geometrical details.

The final change-detection map is generated according to the following iterative procedure, which includes evaluating the optimal number of preprocessing filtering iterations:

Step 1) Initialize $k=1$ and set $N_{\max }$ (maximum number of filtering iterations);

Step 2) Generate $X_{1}^{k}$ and $X_{2}^{k}$ by filtering $X_{1}^{k-1}$ and $X_{2}^{k-1}$, respectively;

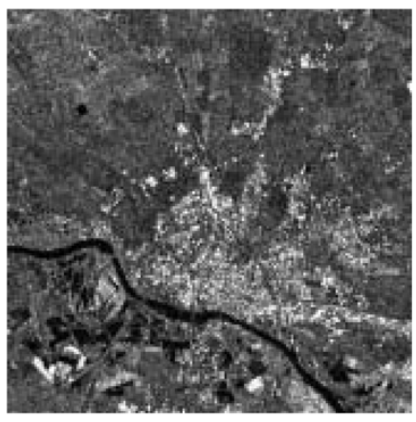

(a)

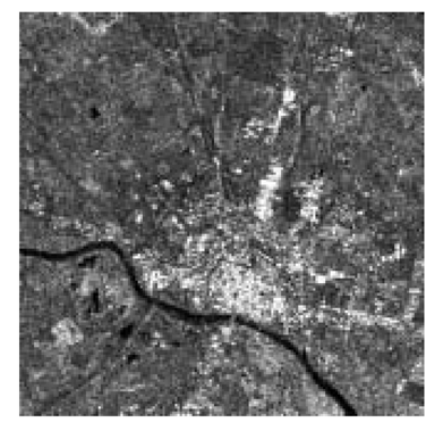

(b)

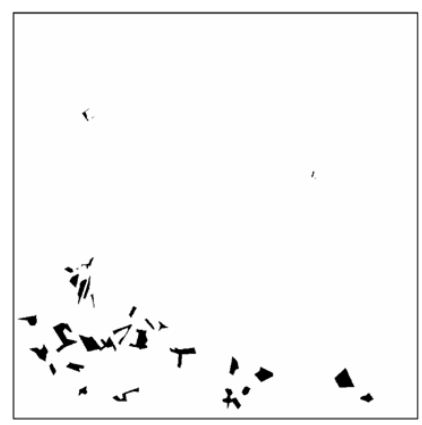

(c)

Fig. 4. Multitemporal images relating to the city of Pavia used in the experiments. (a) Image acquired on October 20, 2000, immediately after the flooding. (b) Image acquired on October 28, 2000 (in this image, most of the flooded areas disappeared). (c) Map of changed areas (ground truth) used as reference in the experiments.

Step 3) Compute the log-ratio image $X_{l}^{k}$;

Step 4) Evaluate the cost function $J^{k}(T)$ and save the corresponding minimum value as the performance index $J(k)$;

Step 5) If $k<N_{\max }$, set $k=k+1$ and go to Step 2);

Step 6) Identify the best number of filtering iterations $k^{*}$ as the one corresponding to the minimum value of $J(k)$;

Step 7) Threshold the log-ratio image $X_{l}^{k^{*}}$ to generate the final change-detection map.

\section{DATASET DEsCRIPTION AND EXPERIMENT DESIGN}

In order to assess the effectiveness of the proposed approach, two datasets with different characteristics and speckle noise levels were considered in the experiments. The first data set represents a region surrounding the city of Pavia, Italy, while the second represents an area near the city of Bern, Switzerland. Detailed descriptions of these datasets and of the experimental activity are provided in the following.

\section{A. Pavia Dataset}

The first dataset used in the experiments is made up of a section $(730 \times 730$ pixels $)$ of two SAR images acquired by the European Remote Sensing 2 satellite SAR sensor. From the two images of the city of Pavia acquired on October 20 and 28, 2000, it is possible to analyze which parts of the area were affected by the flooding that occurred just before the first acquisition date. The ENL of the two images computed according to [28] is 2.64 and 3.43, respectively. This means that the images related to this area are strongly corrupted by speckle noise. Accordingly, this 


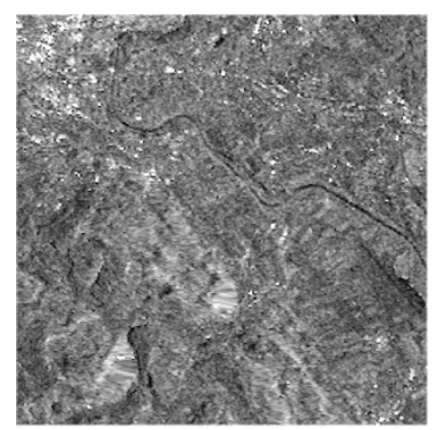

(a)

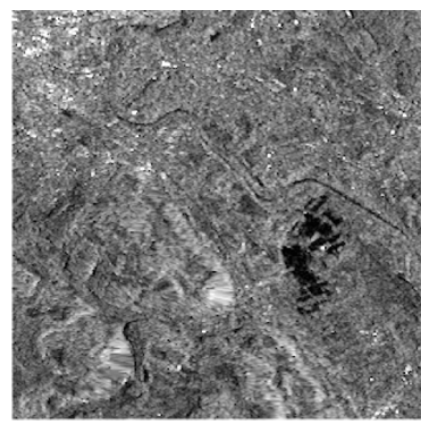

(b)

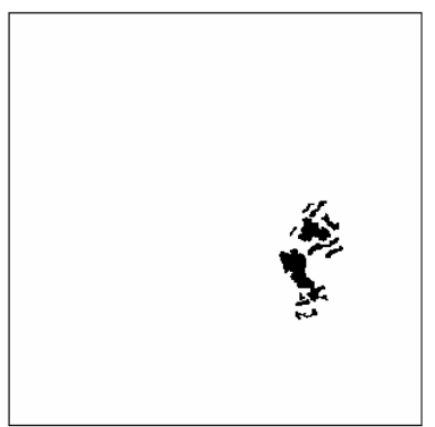

(c)

Fig. 5. Multitemporal images relating to the city of Bern used in the experiments. (a) Image acquired in April 1999 before the flooding. (b) Image acquired in May 1999 after the flooding. (c) Map of changed areas (ground truth) used as reference in the experiments.

is a proper dataset for assessing the effectiveness of the iterative controlled filtering technique integrated in the proposed approach. The images and the ground truth are shown in Fig. 4.

\section{B. Bern Dataset}

The second dataset represents a section $(301 \times 301$ pixels $)$ of two SAR images acquired by the European Remote Sensing 2 satellite SAR sensor over an area near the city of Bern, Switzerland, in April and May 1999, respectively. The ENL of the two small images considered is 10.89 and 9.26, respectively. These high ENL values are explained by the fact that each of the two images was obtained by averaging a pair of Tandem SAR images taken with different viewing angles and by resampling and geocoding the five-look images. Between the two acquisition dates, the river Aare flooded parts of the cities of Thun and Bern and the airport of Bern entirely. Therefore, the Aare valley between Bern and Thun was selected as a test site for detecting flooded areas. The images and the available ground truth (which is obtained by integrating prior information with photo interpretation) are shown in Fig. 5.

\section{Description of the Experiments}

In order to assess the effectiveness of the proposed changedetection approach, four main experiments have been carried out aimed at analyzing: 1) the effects of filtering on the class distributions; 2) the suitability of the Gaussian and generalized Gaussian functions for modeling the statistical distributions of the changed and unchanged classes; 3 ) the effectiveness of the

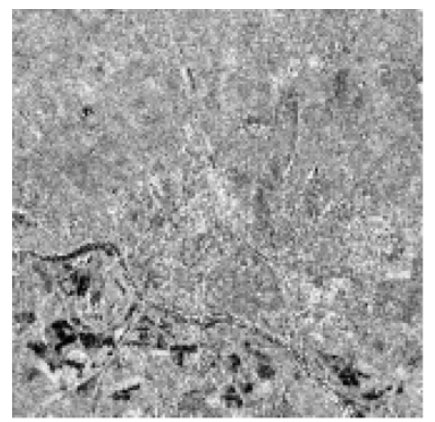

(a)

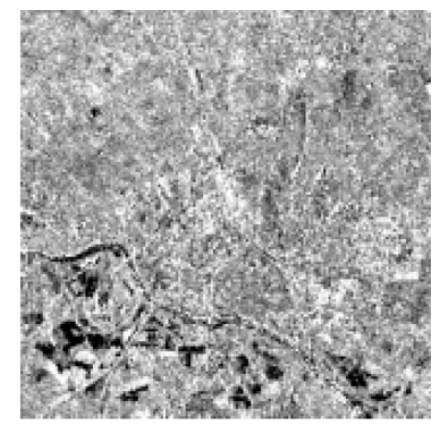

(b)
Fig. 6. Log-ratio images relating to the Pavia dataset generated from (a) original images and (b) filtered images (seven iterations).

automatic threshold selection procedure; 4) the usefulness of the iterative controlled filtering technique.

The first experiment was aimed at evaluating the behavior of the distributions of changed and unchanged classes in the log-ratio images generated from original and filtered images, respectively. In particular, we analyzed the effects of filtering on the statistical distributions of these classes. The analysis of the distributions of changed and unchanged classes in the logratio images was carried out in a supervised way, i.e., using the available ground truth. The filtered images were obtained by an iterative application of the adaptive enhanced Lee filter to the original images using a window size of $3 \times 3$ pixels.

In the second experiment, we assessed the appropriateness of the Gaussian and GG models to estimate the pdfs of changed and unchanged classes studied in the previous experiment. In particular, we used the statistical Kolmogorov-Smirnov (KS) test to evaluate quantitatively the effectiveness of these models in approximating the distributions of the two considered classes before and after despeckling. It is worth noting that the KS test is based on a comparison of the cumulative distributions resulting from the data and the analyzed model. It consists of computing a significance level (probability) $P_{\mathrm{KS}}$, defined according to the maximum value of the absolute difference between the two cumulative distribution functions. A small $P_{\mathrm{KS}}$ value indicates that the estimated model is not suitable to describe the data statistics.

In the third experiment, the effectiveness of the proposed automatic approach to unsupervised threshold selection is assessed in terms of false alarms (i.e., unchanged pixels wrongly classified as changed), detected changes (i.e., changed pixels correctly classified as changed), and overall error (i.e., false and missed alarms). The results obtained on the different log-ratio images (generated from the two multitemporal original images with different values of $k$, i.e., different filtering iterations) by selecting the threshold automatically with the assumptions of both Gaussian and GG distributions were compared with those yielded by the minimum error threshold selected according to a supervised manual trial-and-error procedure (MTEP). To this end, the minimum-error threshold was derived by performing a nonautomatic evaluation of the overall change-detection errors versus all the possible values of the decision threshold; then the threshold value that yielded the minimum overall error was chosen.

Finally, the fourth experiment is intended to assess the suitability of the cost function $J(k)$ as a performance index for the 


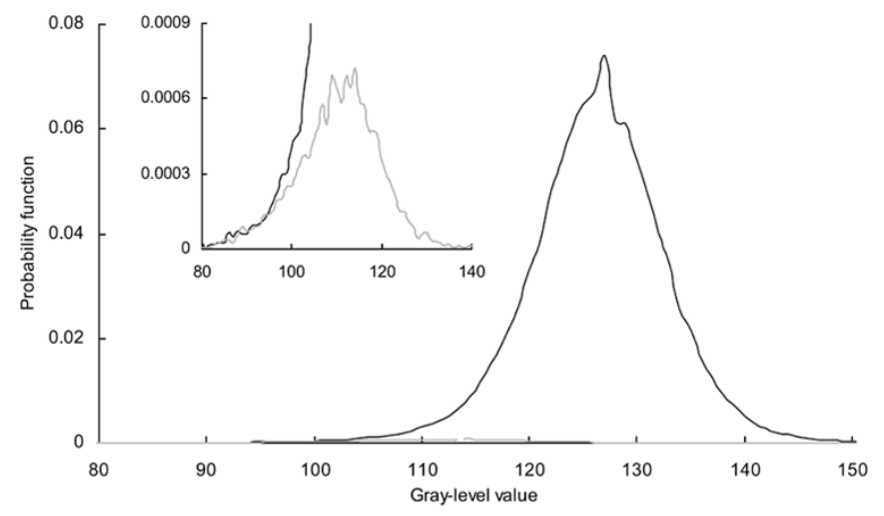

(a)

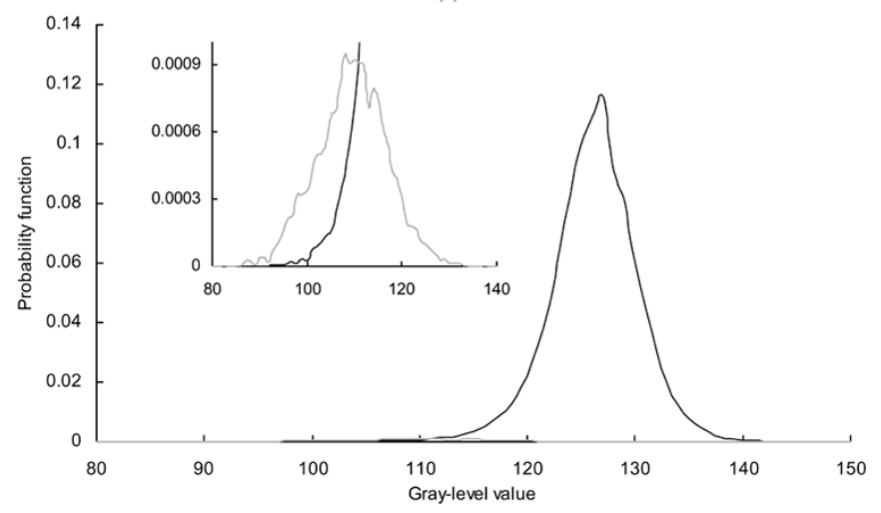

(b)

Fig. 7. Distributions of changed and unchanged classes in the log-ratio images of the Pavia dataset generated from (a) original images and (b) filtered images (seven iterations). Thanks to the zoom of the histogram, it is possible to observe the distribution of the changed class (faint line) whose prior probability is significantly lower than that of the unchanged class (thick line).

selection of the best number of filtering iterations required to generate the change-detection map with the least overall error.

\section{EXPERIMENTAL RESULTS}

\section{A. Results Obtained on the Pavia Dataset}

In the first experiment, we analyzed the distributions of the changed and unchanged classes in the log-ratio images generated from the original and filtered images (Fig. 6), respectively. Fig. 7 shows these distributions extracted according to the use of the ground-truth information. As expected from the estimated ENL (i.e., 2.64 and 3.43), in this dataset changed and unchanged classes in the log-ratio image generated from the original multitemporal images [Fig. 6(a)] were almost completely overlapped [Fig. 7(a)]. This depends on the fact that these images are highly corrupted by speckle noise, which makes it difficult and unreliable to use any unsupervised change-detection method based on the analysis of the log-ratio image (it is not possible to distinguish the two classes with a simple thresholding procedure). In order to separate these two classes, several filtering iterations were applied to the original images. As an example, Fig. 7(b) and Table I show the significant improvement in separation between the two considered classes in the log-ratio image obtained after filtering the original images seven times [see Fig. 6(b)].

In the second experiment, the Gaussian and the GG parametric distributions of the changed and unchanged classes were estimated using the available ground truth. Both models
TABLE I

Mean and Standard Deviation Values Related to Changed AND UNCHANGED CLASSES COMPUTED USING THE AVAILABLE GROUND TRUTH ON THE LOG-RATIO IMAGES GENERATED From THE ORIGINAL AND FILTERED Multitemporal IMAGES (SEVEN FILTERING ITERATIONS) (PAVIA DATASET)

\begin{tabular}{l|c|c|c|c}
\cline { 2 - 5 } & \multicolumn{2}{|c|}{ Changed Class } & \multicolumn{2}{c}{ Unchanged Class } \\
\cline { 2 - 5 } & Mean & Stdv & Mean & Stdv \\
\hline Before filtering & 114 & 35 & 125 & 13 \\
\hline After filtering & 106 & 12 & 125 & 7 \\
\hline
\end{tabular}

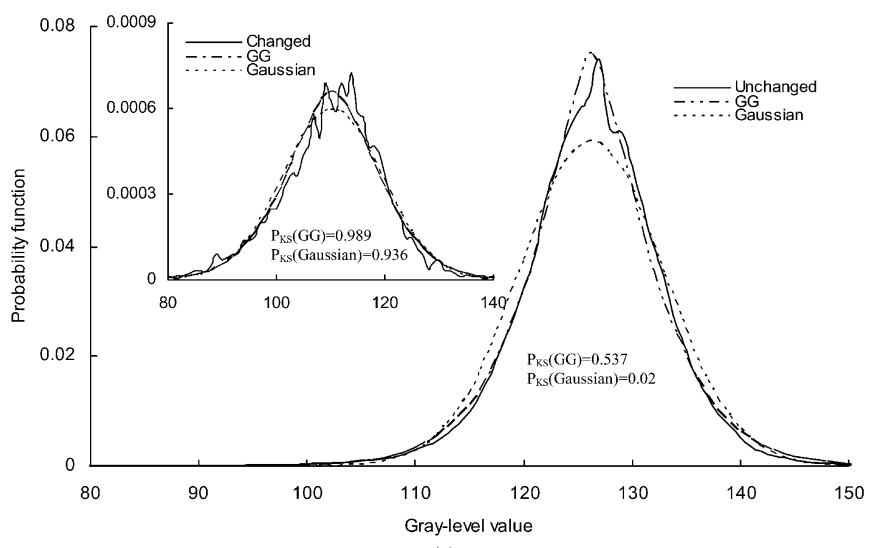

(a)

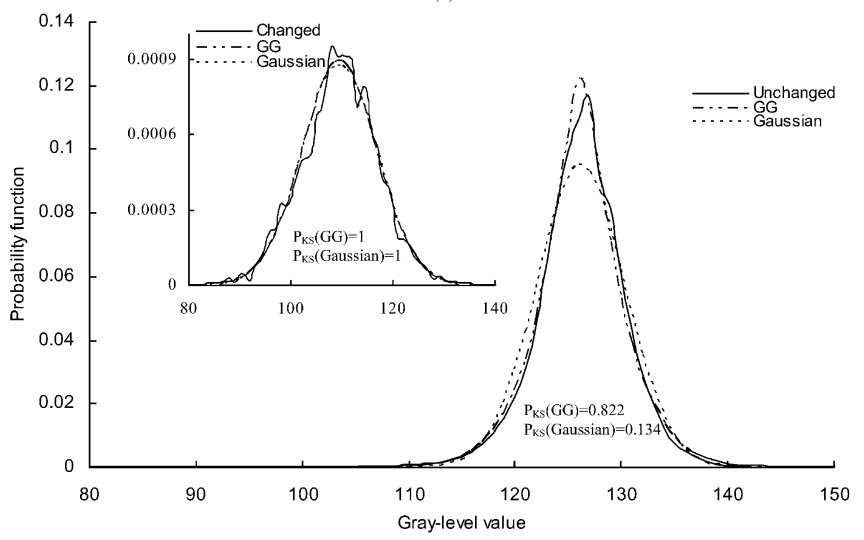

(b)

Fig. 8. Comparison between the Gaussian and GG models for approximating the distributions associated with changed and unchanged classes in the log-ratio images of the Pavia dataset generated from (a) original images and (b) filtered images (seven filtering iterations). In both figures, the zoom shows the two models together with the true distribution of the changed class.

required the estimation of the mean and variance values. In addition, in the case of the GG model, it was necessary to estimate the shape parameter $(\beta)$ according to the procedure described in Section III-B. It is worth recalling that the use of ground truth is aimed at driving the statistical analysis of the changed and unchanged classes in the first two experiments in order to: 1) understand the effect of filtering on class distributions; and 2) to evaluate the goodness-of-fit of each of the two investigated parametric models. However, the change-detection process described in the next two experiments is carried out in a completely unsupervised way (i.e., assuming that no reference data are available). As one can see from Fig. 8(a), the two models did not provide an accurate description of the two classes before despeckling. This is confirmed by the KS test, which resulted in small $P_{\mathrm{KS}}$ values for both functions. On the 


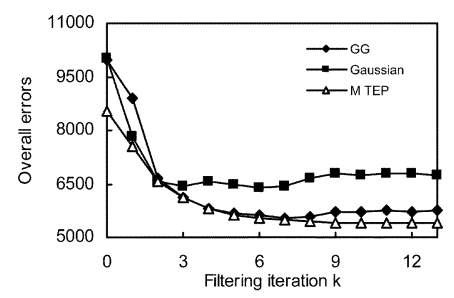

(a)

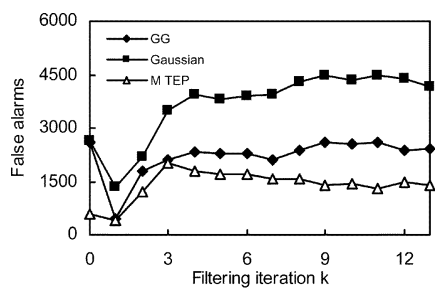

(b)

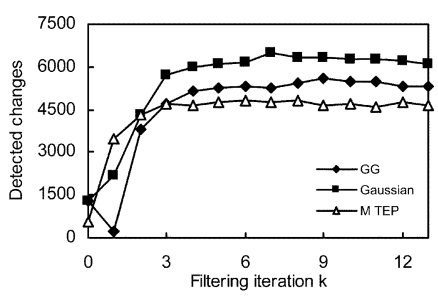

(c)
Fig. 9. Change-detection results (reported in number of pixels) obtained with the automatic threshold selection procedure (under the Gaussian and the GG assumptions) and the reference MTEP versus the number of filtering iterations (Pavia dataset). (a) Overall error. (b) False alarms. (c) Detected changes.

other hand, as expected, a better approximation was obtained after filtering the original images seven times [Fig. 8(b)]. In this case, the two considered models resulted in different approximations of the class distributions. According to the $P_{\mathrm{KS}}$ values, better approximations were obtained under the GG assumption (especially for the unchanged class), confirming the better fitting properties of this model compared to the Gaussian.

In the third experiment, the thresholding of the log-ratio image generated from the original multitemporal images resulted in high overall errors for both models (i.e., 10028 and 9981 misclassified pixels for the Gaussian and GG distributions, respectively). This is explained by the almost complete overlap between the two class distributions in the log-ratio image, as observed in the first experiment. By contrast, by filtering the original images several times, the change-detection accuracy improved significantly. This is illustrated in Fig. 9, which shows the behavior of the overall error, false alarms, and detected changes versus the number of filtering iterations. Compared with what was obtained with the Gaussian model, the change-detection accuracies achieved under the GG assumption were closer to those obtained by the reference MTEP. This is explained by the limited capability of the Gaussian distribution to model the changed and unchanged classes in the log-ratio image; this confirmed the results obtained by the KS test in the previous experiment. According to Fig. 9, for both distributions the optimal change-detection result in terms of overall error was obtained after filtering the original multitemporal images seven times.

In the fourth experiment, we assessed the effectiveness of the proposed procedure for the unsupervised identification of the best filtering iteration number. This procedure is equivalent to selecting the optimal change-detection map using the cost function $J(k)$ (computed over the number of filtering iterations) as a performance index. Fig. 10 clearly shows that according to the value of $J(k)$, the best change-detection accuracy for both considered models was estimated with seven filtering iterations. This corresponds to what was observed in a supervised way in the previous experiment, i.e., the change-detection map with

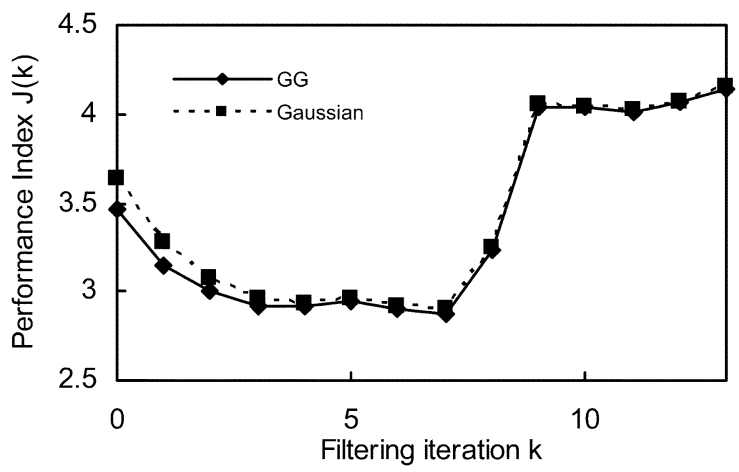

Fig. 10. Behavior of the cost function $J(k)$ versus number of filtering iterations (Pavia dataset).

TABLE II

RESUlts ACHIEVED BY THE PROPOSED APPROACH (UNDER THE GAUSSIAN AND GG ASSUMPTIONS) AND THE MTEP (PAVIA DATASET)

\begin{tabular}{c||c|c|c|c} 
& Threshold Value T & Overall Error & False Alarms & Detected Changes \\
\hline \hline Gaussian & 114 & 6423 & 3950 & 6512 \\
\hline GG & 111 & 5550 & 2116 & 5235 \\
\hline MTEP & 110 & 5483 & 1568 & 4751 \\
\hline
\end{tabular}

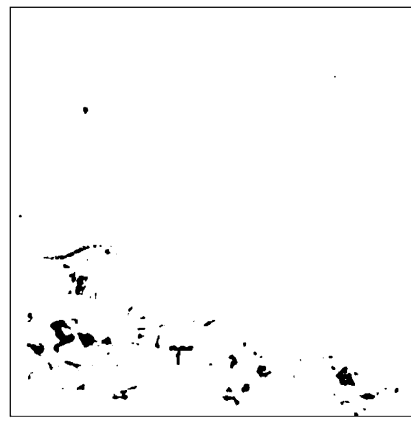

(a)

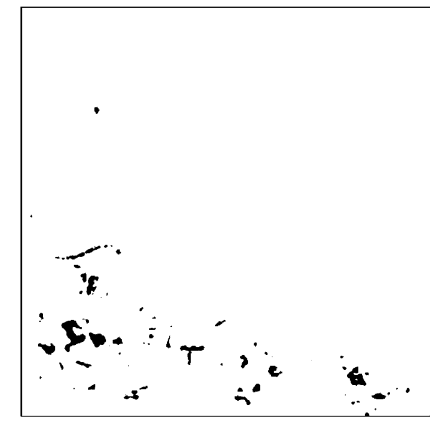

(b)
Fig. 11. Final change-detection maps obtained from the analysis of the log-ratio image relating to the Pavia dataset by (a) the proposed approach under the GG assumption and (b) MTEP.

the minimum overall error is achieved after applying seven iterations of the filter to the original images. In this case, as reported in Table II, the threshold value under the Gaussian assumption was equal to 114 (corresponding to an overall error of 6423 pixels, 3950 false alarms, and 6512 changed pixels correctly detected), while it was 111 under the GG assumption (corresponding to an overall error of 5550 pixels, 2116 false alarms, and 5235 changed pixels correctly detected). The threshold value obtained by the nonautomatic MTEP was 110 (corresponding to an overall error of 5483 pixels, 1568 false alarms, and 4751 changed pixels correctly detected). This quantitative comparison confirms the effectiveness of the proposed approach based on the GG parametric model. This is also shown by the change-detection maps (Fig. 11) obtained both by the proposed approach and by the MTEP, which are very similar to each other and very close to the ground-truth map depicted in Fig. 4(c).

\section{B. Results Obtained on the Bern Dataset}

Unlike the previous dataset, the multitemporal Bern SAR images are characterized by high ENL values. This represents 

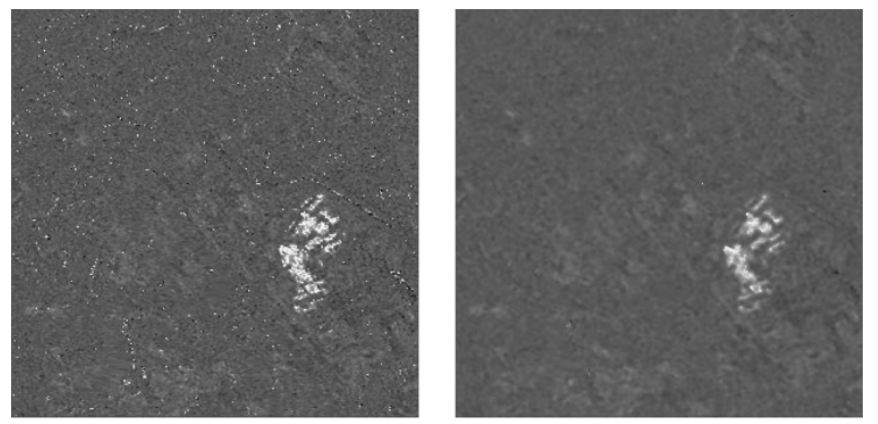

(a)

(b)

Fig. 12. Log-ratio images of the Bern dataset generated from (a) original images and (b) filtered images (one filtering iteration).

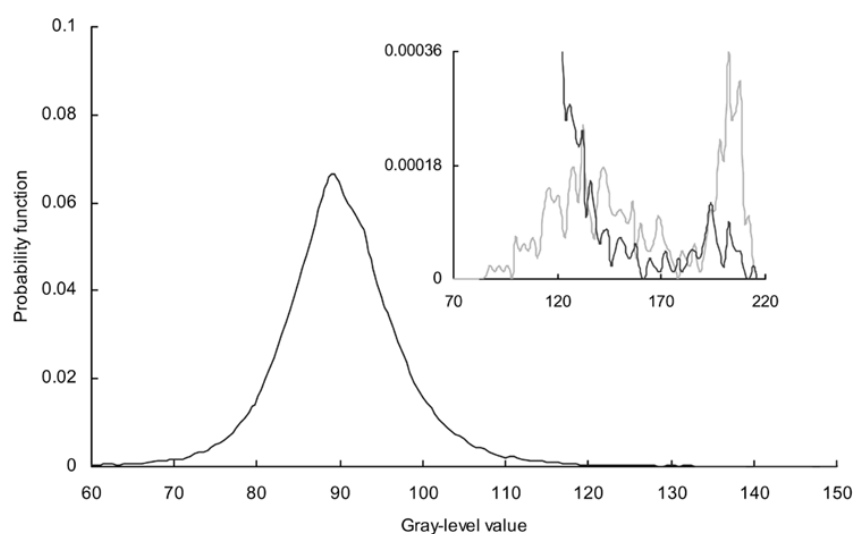

(a)

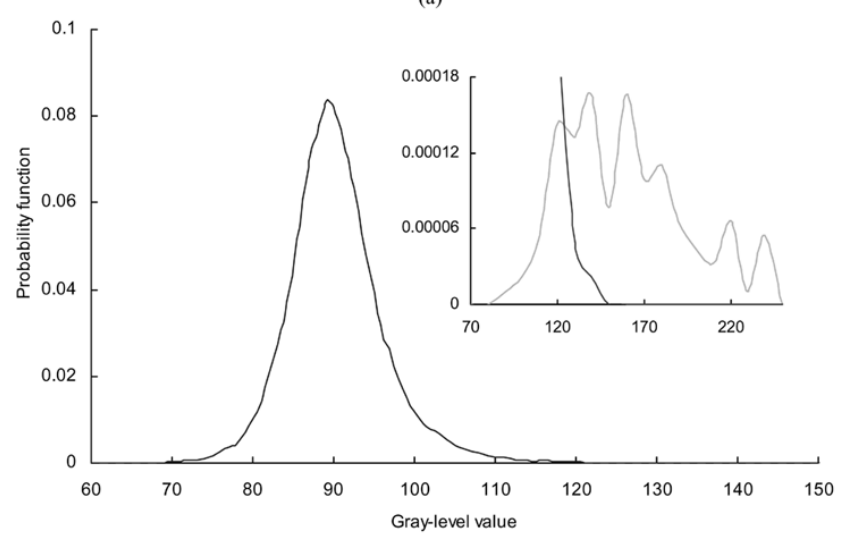

(b)

Fig. 13. Distributions of changed and unchanged classes in the log-ratio images of the Bern dataset generated from (a) original images and (b) filtered images (one filtering iteration). Thanks to the zoom of the histogram, it is possible to observe the distribution of the changed class (faint line), whose prior probability significantly lower than that of the unchanged class (thick line).

a completely different benchmark to assess the effectiveness of the proposed approach (and in particular of the controlled adaptive iterative filtering procedure presented, since the images given as input to our system are already affected by a low noise level).

In the first experiment related to the analysis of the distributions of changed and unchanged classes in the log-ratio image (see Fig. 12), the changed class resulted in the presence of two modes very close together [see Fig. 13(a)]. This is explained by the fact that the flooding occurred mainly on two different ground covers characterized by different backscattering values.

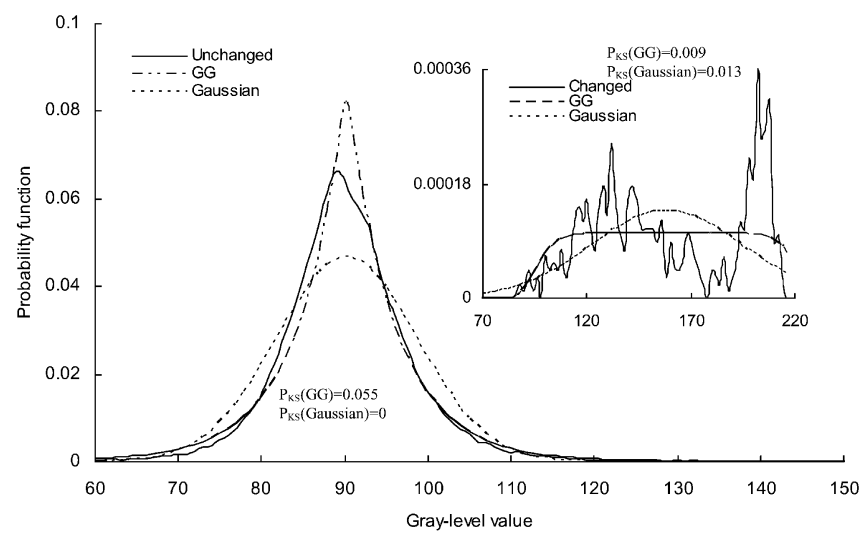

(a)

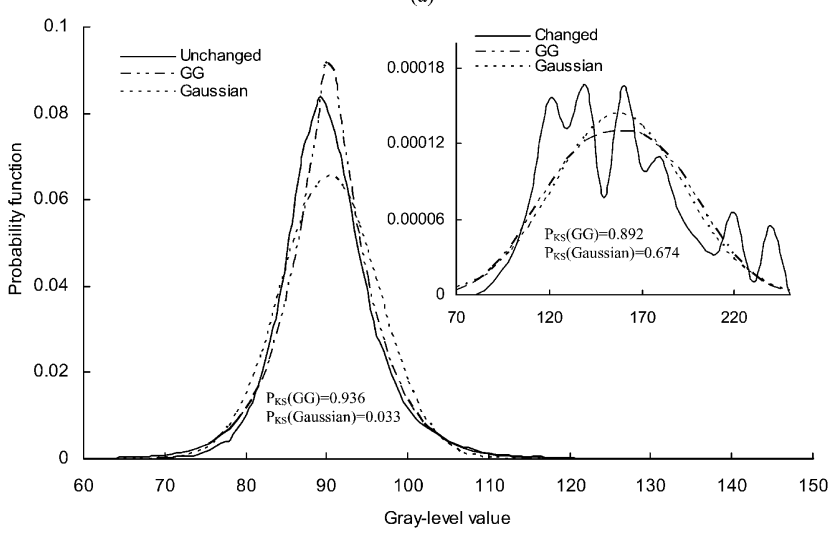

(b)

Fig. 14. Comparison between the Gaussian and the GG models to approximate the distributions associated with changed and unchanged classes in the log-ratio images of the Bern dataset generated from (a) original images and (b) filtered images (one iteration). In both figures, the zoom shows the two models together with the true distribution of the changed class.

In this case, according to the higher ENL values characterizing the two images, the changed and unchanged classes in the data given as input to the proposed system overlap less than in the Pavia dataset. However, as shown in Fig. 13(b), the application of one iteration of the enhanced Lee filter improved class separability further by decreasing the overlap that characterizes two-class conditional distributions.

In the second experiment, as shown in Fig. 14, the Gaussian and GG models resulted in different approximations of the distribution of changed and unchanged classes in the log-ratio images obtained without and with filtering. Similarly to what found in the Pavia dataset, the $P_{\mathrm{KS}}$ values confirm the superiority of the GG model over the Gaussian.

In the third experiment, we first ran the procedure for automatic threshold selection on the log-ratio image generated from original multitemporal images. The obtained change detection overall errors were equal to 715 and 776 pixels, for the GG and the Gaussian distributions, respectively. We then applied this procedure to a set of log-ratio images generated from the filtered images by varying the number of filtering iterations from 1 to 4 (we did not vary the number of iterations much, as the ENL of the original images was already high). The obtained results were significantly different for the two models. The behaviors of the overall error, false alarms, and detected changes versus the number of filtering iterations are shown in Fig. 15. As can be seen, unlike the Gaussian case, the change-detection accuracy 


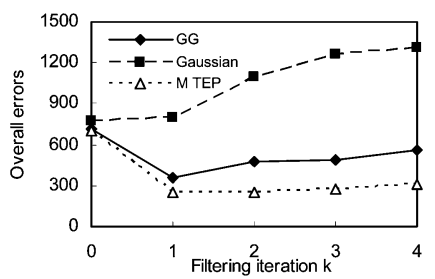

(a)

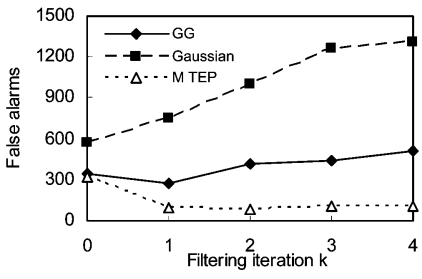

(b)

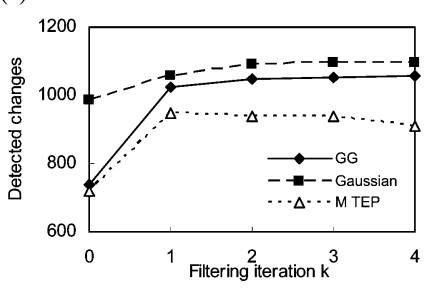

(c)
Fig. 15. Change-detection results (reported in number of pixels) obtained with the automatic threshold selection procedure (under the Gaussian and the GG assumptions) and the reference MTEP versus the number of filtering iterations (Bern dataset). (a) Overall error. (b) False alarms. (c) Detected changes.

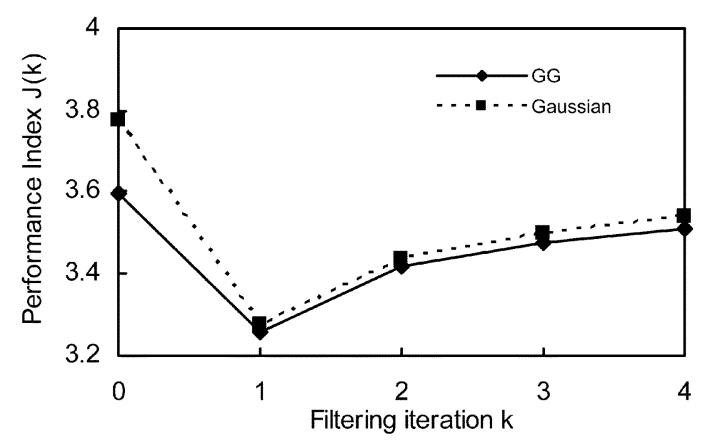

Fig. 16. Behavior of the cost function $J(k)$ versus number of filtering iterations (Bern dataset).

TABLE III

Results AchiEved By the Proposed APPROACH (UNDER THE GAUSSIAN AND GG ASSUMPTIONS) AND THE MTEP (BERN DATASET)

\begin{tabular}{c|c|c|c|c} 
& Threshold Value T & Overall Error & False Alarms & Detected Changes \\
\hline \hline Gaussian & 109 & 803 & 755 & 1057 \\
\hline GG & 115 & 360 & 277 & 1022 \\
\hline MTEP & 123 & 252 & 93 & 946 \\
\hline
\end{tabular}

obtained under the GG model was close to that obtained with the optimal manual trial-and-error procedure (MTEP). This again confirms the superiority of the more general GG model over the Gaussian.

In the fourth experiment, the unsupervised identification of the optimal number of filtering iterations based on the performance index $J(k)$ confirmed what was observed in the previous dataset. In fact, according to the behavior of $J(k)$ (Fig. 16), the best number of filtering iterations was equal to one. This result corresponds to what was found by the trial-and-error procedure in the previous experiment. It is worth noting that this points out the accuracy and sensitivity of the proposed controlled adaptive filtering procedure, which proved to be very effective also in presence of images affected by a low noise level. In this case, as reported in Table III, the threshold value obtained under the Gaussian assumption was equal to 109 (corresponding to an overall error of 803 pixels, 755 false alarms, and 1057 changed

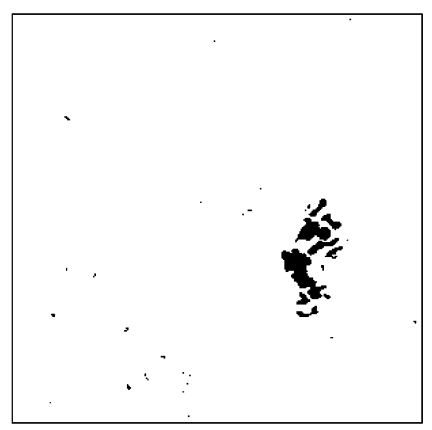

(a)

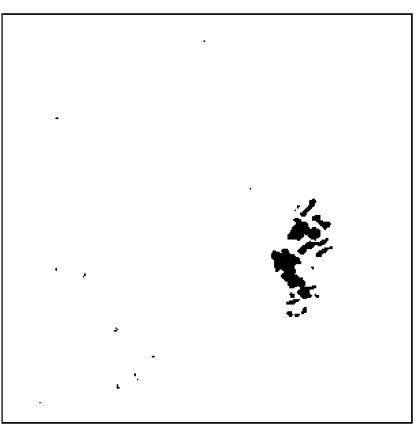

(b)
Fig. 17. Final change-detection maps obtained from the analysis of the log-ratio image relating to the Bern dataset by (a) the proposed approach under the GG assumption and (b) MTEP.

pixels correctly detected), while it was 115 under the GG assumption (corresponding to an overall error of 360 pixels, 277 false alarms, and 1022 changed pixels correctly detected). On the other hand, the threshold value obtained by the MTEP was $T=123$ (corresponding to an overall error of 252 pixels, 93 false alarms, and 946 changed pixels correctly detected). These results reveal that the proposed automatic change-detection approach achieved accuracies very close to those exhibited by the supervised MTEP, which of course provides the best possible results. The final change-detection maps obtained by these two methods are shown in Fig. 17. A visual comparison of these two maps shows that they are very similar to each other and close to the ground-truth image [Fig. 5(c)].

\section{DISCUSSION AND CONCLUSION}

In this paper, a novel unsupervised change-detection approach specifically oriented to the analysis of single-channel single-polarization multitemporal SAR images has been presented. This approach is based on an automatic analysis of the log-ratio image (generated from filtered multitemporal SAR images) carried out by proposing an extension of the KI threshold selection algorithm. Two novel methodological contributions characterize this work compared to traditional unsupervised change-detection techniques: 1) the optimal threshold value is determined in a completely automatic way; and 2) the optimal number of despeckling filtering iterations (with respect to the expected change-detection error) applied to the original images in the preprocessing step is estimated automatically. As regards the first contribution, the KI algorithm (which was originally developed for the Gaussian case) has been extended to SAR images by reformulating it under the GG assumption for the changed and unchanged classes. This model is attractive, because it can approximate a large variety of statistical distributions at the cost of only one additional parameter to be estimated (i.e., the shape parameter). The second contribution consists of exploiting the KI criterion to estimate the optimal number of filtering iterations that results in the highest expected change-detection accuracy.

The experimental results obtained on two different multitemporal SAR images confirmed the effectiveness of the proposed approach. In particular, as expected, the GG distribution model proved to be more suitable than the Gaussian one to fit the distributions of unchanged and changed classes. In addition, with the 
presented controlled adaptive iterative filtering procedure, it was possible to identify in an unsupervised and automatic way the "optimal" number of iterations to be considered in the despeckling phase (it is worth noting that the term "optimal" is used with respect to the considered statistical model implemented in the adopted change-detection approach). This avoids the problems associated with the wrong number of iterations of the filtering process, which may affect the accuracy of the change-detection process (after a given iteration number, the iterative filtering process may decrease the accuracy of the change-detection process by significantly losing details present in the images). The aforementioned procedure proved suitable in two situations characterized by low and high ENL values in the original multitemporal SAR images. The results in terms of overall error, false alarms, and detected changes confirmed that the proposed approach can achieve performances very close to the optimal ones exhibited by the reference supervised MTEP.

It is worth noting that the proposed approach can be used with any adaptive speckle filter. The adoption of filters with different effectiveness will result in the identification of different numbers of "optimal" iterations and therefore in a different tradeoff between accuracy in homogeneous areas and the preservation of geometrical details.

On the one hand, the main advantages of the proposed approach are: 1) its computational simplicity (the most time-consuming phase is related to speckle filtering); 2) its stability (i.e., it does not depend on initial conditions); and 3) its generality with respect to the typology of changes occurred on the ground (thanks to the fact that it uses a flexible statistical model such as GG distribution). Another interesting feature is the possibility of generalizing it in order to identify one or more thresholds in the log-ratio image, depending on the presence of one or more change typologies between the two acquisition dates (e.g., presence of changes on both sides of the histogram of the log-ratio image). On the other hand, its main drawbacks are that: 1) it does not take full advantage of all the information present in the speckle (the iterative filtering also reduces the amount of information present in the speckle); 2) it is more focused on the thresholding task rather than on the correct estimation of changed and unchanged class statistics (whose implicit estimations prove to be biased). The latter issue does not allow to exploit more sophisticated decision strategies, such as those based on the use of spatial contextual information, for the definition of the change-detection map [6], [8]. This aspect will be studied as a future development of this work.

\section{APPENDIX}

In this appendix, we derive (14), which expresses the KI selection criterion assuming a GG distribution model for the changed and unchanged classes. Under this assumption, the cost function $c\left(X_{l}, T\right)$ defined by the $\mathrm{KI}$ algorithm is given by

$$
c\left(X_{l}, T\right)=\left\{\begin{array}{cc}
-2 \ln \left[P_{u}(T) a_{u}(T)\right. & \\
\left.\times e^{-\left[b_{u}(T)\left|X_{l}-m_{u}(T)\right|\right]^{\beta_{u}(T)}}\right], & \text { if } X_{l} \leq T \\
-2 \ln \left[P_{c}(T) a_{c}(T)\right. & \\
\left.\times e^{-\left[b_{c}(T)\left|X_{l}-m_{c}(T)\right|\right]^{\beta_{c}(T)}}\right], & \text { if } X_{l}>T .
\end{array}\right.
$$

Equation (15) can be rewritten as follows:

$c\left(X_{l}, T\right)=\left\{\begin{array}{cc}2\left[b_{u}(T)\left|X_{l}-m_{u}(T)\right|\right]^{\beta_{u}(T)} & \\ -2 \ln a_{u}(T)-2 \ln P & \\ 2\left[b_{c}(T)\left|X_{l}-m_{c}(T)\right|\right]^{\beta_{c}(T)} & \text { if } X_{l} \leq T \\ -2 \ln a_{c}(T)-2 \ln P_{c}(T), & \text { if } X_{l}>T\end{array}\right.$

Substituting (16) in (3), we obtain (17), shown at the bottom of the page. Substituting $P_{u}(T)=\sum_{X_{l}=0}^{T} h\left(X_{l}\right)$ and $P_{c}(T)=$ $\sum_{X_{l}=T+1}^{L-1} h\left(X_{l}\right)$ in (17) and removing the common multiplicative factor, we obtain the following final form of the cost function $J(T)$ for threshold selection:

$$
\begin{aligned}
J(T)= & \sum_{X_{l}=0}^{T} h\left(X_{l}\right)\left[b_{u}(T)\left|X_{l}-m_{u}(T)\right|\right]^{\beta_{u}(T)} \\
& +\sum_{X_{l}=T+1}^{L-1} h\left(X_{l}\right)\left[b_{c}(T)\left|X_{l}-m_{c}(T)\right|\right]^{\beta_{c}(T)} \\
& +H(\Omega, T)-\left[P_{u}(T) \ln a_{u}(T)\right. \\
& \left.+P_{c}(T) \ln a_{c}(T)\right]
\end{aligned}
$$

where $H(\Omega, T)$ is the entropy associated with the binary set of classes $\Omega=\left\{\omega_{c}, \omega_{u}\right\}$, which is defined as follows:

$$
H(\Omega, T)=-\sum_{X_{l}=0}^{T} h\left(X_{l}\right) \ln P_{u}(T)-\sum_{X_{l}=T+1}^{L-1} h\left(X_{l}\right) \ln P_{c}(T) .
$$

$$
\begin{aligned}
J(T)= & 2 \sum_{X_{l}=0}^{T} h\left(X_{l}\right)\left\{\left[b_{u}(T)\left|X_{l}-m_{u}(T)\right|\right]^{\beta_{u}(T)}-\ln a_{u}(T)-\ln P_{u}(T)\right\}+2 \sum_{X_{l}=T+1}^{L-1} h\left(X_{l}\right)\left\{\left[b_{c}(T)\left|X_{l}-m_{c}(T)\right|\right]^{\beta_{c}(T)}\right. \\
& \left.-\ln a_{c}(T)-\ln P_{c}(T)\right\} \\
= & 2 \sum_{X_{l}=0}^{T} h\left(X_{l}\right)\left[b_{u}(T)\left|X_{l}-m_{u}(T)\right|\right]^{\beta_{u}(T)}+2 \sum_{X_{l}=T+1}^{L-1} h\left(X_{l}\right)\left[b_{c}(T)\left|X_{l}-m_{c}(T)\right|\right]^{\beta_{c}(T)}-2 \sum_{X_{l}=0}^{T} h\left(X_{l}\right) \ln a_{u}(T)-2 \\
& \times \sum_{X_{l}=T+1}^{L-1} h\left(X_{l}\right) \ln a_{c}(T)-2 \sum_{X_{l}=0}^{T} h\left(X_{l}\right) \ln P_{u}(T)-2 \sum_{X_{l}=T+1}^{L-1} h\left(X_{l}\right) \ln P_{c}(T) .
\end{aligned}
$$




\section{ACKNOWLEDGMENT}

The authors wish to thank U. Wegmuller (Gamma Remote Sensing, Bern, Switzerland) and P. Gamba (University of Pavia, Pavia, Italy) for providing the multitemporal SAR images used in the experiments.

\section{REFERENCES}

[1] P. S. Chavez and D. J. Mackinnon, "Automatic detection of vegetation changes in the southwestern united states using remotely sensed images," Photogramm. Eng. Remote Sens., vol. 60, pp. 1285-1294, 1994.

[2] L. Bruzzone and S. B. Serpico, "An iterative technique for the detection of land-cover transitions in multispectral remote-sensing images," IEEE. Trans. Geosci. Remote Sens., vol. 35, no. 4, pp. 858-867, Jul. 1997.

[3] K. R. Merril and L. Jiajun, "A comparison of four algorithms for change detection in an urban environment," Remote Sens. Environ., vol. 63, pp. 95-100, 1998.

[4] T. Hame, I. Heiler, and J. S. Miguel-Ayanz, "An unsupervised change detection and recognition system for forestry," Int. J. Remote Sens., vol. 19, pp. 1079-1099, 1998.

[5] A. Singh, "Digital change detection techniques using remotely sensed data," Int. J. Remote Sens., vol. 10, pp. 989-1003, 1989.

[6] L. Bruzzone and D. F. Prieto, "Automatic analysis of the difference image for unsupervised change detection," IEEE Trans. Geosci. Remote Sens., vol. 38, no. 3, pp. 1171-1182, May 2000.

[7] P. Dempster, N. M. Laird, and D. B. Rubin, "Maximum likelihood from incomplete data via the EM algorithm," J. R. Stat. Soc., vol. 39, pp. 1-38, 1977.

[8] L. Bruzzone and D. F. Prieto, "An adaptive and semiparametric and context-based approach to unsupervised change-detection in multitemporal remote sensing images," IEEE Trans. Image Process., vol. 11, no. 4, pp. 452-466, Apr. 2002.

[9] T. Kasetkasem and P. K. Varshney, "An image change-detection algorithm based on Markov random filed models," IEEE Trans. Geosci. Remote Sens., vol. 40, no. 8, pp. 1815-1823, Aug. 2002.

[10] F. Melgani, G. Moser, and S. B. Serpico, "Unsupervised change-detection methods for remote-sensing data," Opt. Eng., vol. 41, pp. 3288-3297, 2002.

[11] J. Cihlar, T. J. Pultz, and A. L. Gray, "Change detection with synthetic aperture radar," Int. J. Remote Sens., vol. 13, pp. 401-414, 1992.

[12] E. J. M. Rignot and J. J. Van Zyl, "Change detection techniques for ERS-1 SAR data," IEEE Trans. Geosci. Remote Sens., vol. 31, no. 4, pp. 896-906, Jul. 1993.

[13] J. D. Villasenor, D. R. Fatland, and L. D. Hinzman, "Change detection on Alaska's north slope using repeat-pass ERS-1 SAR imagery," IEEE Trans. Geosci. Remote Sens., vol. 31, pp. 227-236, 1993.

[14] R. J. Dekker, "Speckle filtering in satellite SAR change detection imagery," Int. J. Remote Sens., vol. 19, pp. 1133-1146, 1998.

[15] K. Grover and S. Quegan, "Quantitative estimation of tropical forest cover by SAR," IEEE Trans. Geosci. Remote Sens., vol. 37, no. 1, pp. 479-490, Jan. 1999.

[16] Y. Moisan, M. Bernier, and J.-M. M. Dubois, "Détection des changements dans une série d'images ERS-1 multidates a l'aide de l'analyze en composante principale," Int. J. Remote Sens., vol. 20, pp. 1149-1167, 1999.

[17] J. E. S. Fransson, F. Walter, K. Blennow, A. Gustavsson, and L. M. H. Ulander, "Detection of storm-damaged forested areas using airborne CARABAS-II VHF SAR image data," IEEE Trans. Geosci. Remote Sens., vol. 40, no. 10, pp. 2170-2175, Oct. 2002.

[18] W. Dierking and H. Skriver, "Change detection for the thematic mapping by means of airborne multitemporal polarimetric SAR imagery," IEEE Trans. Geosc. Remote Sens., vol. 40, no. 3, pp. 618-636, Mar. 2002.

[19] F. T. Bujor, J.-M. Nicolas, E. Trouvé, and J.-P. Rudant, "Application of log-cumulants to change detection in multi-temporal SAR images," in Proc. IGARSS, vol. 2, Toulouse, France, 2003, pp. 1386-1388.

[20] J. Kittler and J. Illingworth, "Minimum error thresholding," Pattern Recognit., vol. 19, pp. 41-47, 1986.

[21] J. S. Lee, "Speckle suppression and analysis for synthetic aperture radar images," Opt. Eng., pp. 636-643, 1986.

[22] D. T. Kuan, A. A. Sawchuk, T. C. Strand, and P. Chavel, "Adaptive noise smoothing filter for images with signal- dependent noise," IEEE Trans. Pattern Anal. Mach. Intell., vol. PAMI-2, pp. 165-177, 1985.
[23] V. S. Frost and J. A. Stiles, "A model for radar images and its application to adaptive digital filtering of multiplicative noise," IEEE Trans. Pattern Anal. Mach. Intell., vol. PAMI-4, pp. 157-166, 1982.

[24] D. T. Kuan, A. A. Sawchuk, T. C. Strand, and P. Chavel, "Adaptive restoration of SAR images with speckle," IEEE Trans. Acoust., Speech, Signal Process., vol. ASSP-35, pp. 373-383, 1987.

[25] A. Lopes, R. Touzi, and E. Nezry, "Adaptive speckle filters and scene heterogeneity," IEEE Trans. Geosci. Remote Sens., vol. 28, no. 6, pp. 992-1000, Nov. 1990

[26] K. Sharifi and A. Leon-Garcia, "Estimation of shape parameter for generalized Gaussian distributions in subband decomposition of video," IEEE Trans. Circuits, Syst. Video Technol., vol. 5, no. 1, pp. 52-56, Feb. 1995.

[27] W. Niehsen, "Generalized Gaussian modeling of correlated signal sources," IEEE Trans. Signal Process., vol. 47, no. 1, pp. 217-219, Jan. 1999.

[28] C. Oliver and S. Quegan, Understanding Synthetic Aperture Radar Images. Boston, MA: Artech House, 1998.

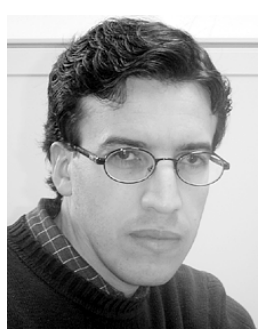

Yakoub Bazi (S'05) received the State Engineer and M.Sc. degrees in electronics from the University of Batna, Batna, Algeria, in 1994 and 2000, respectively. He is currently purusing the Ph.D. degree at the University of Trento, Trento, Italy.

From 2000 to 2002, he was a Lecturer with the University of M'sila, M'sila, Algeria. His current research interests revolve around pattern recognition methodologies and evolutionary computation in multitemporal remote sensing image analysis (change detection, classification, and data fusion)

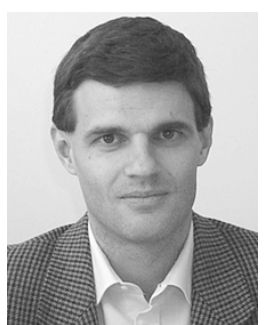

Lorenzo Bruzzone (S'95-M'99-SM'03) received the laurea (M.S.) degree in electronic engineering (summa cum laude) and the Ph.D. degree in telecommunications from the University of Genoa, Genoa, Italy, in 1993 and 1998, respectively.

He is currently Head of the Remote Sensing Laboratory in the Department of Information and Communication Technologies at the University of Trento, Trento, Italy. From 1998 to 2000, he was a Postdoctoral Researcher at the University of Genoa. From 2000 to 2001, he was an Assistant Professor at the University of Trento, and from 2001 to February 2005 he was an Associate Professor of telecommunications at the same university. Since March 2005, he has been a Full Professor of telecommunications at the University of Trento, where he currently teaches remote sensing, pattern recognition, and electrical communications. His current research interests are in the area of remote sensing image processing and recognition (analysis of multitemporal data, feature selection, classification, regression, data fusion, and neural networks). He conducts and supervises research on these topics within the frameworks of several national and international projects. He is the author (or coauthor) of more than 130 scientific publications, including journals, book chapters, and conference proceedings. He is a referee for many international journals and has served on the Scientific Committees of several international conferences. He is a member of the Scientific Committee of the India-Italy Center for Advanced Research.

Dr. Bruzzone ranked first place in the Student Prize Paper Competition of the 1998 IEEE International Geoscience and Remote Sensing Symposium (Seattle, July 1998). He was a recipient of the Recognition of IEEE Transactions on Geoscience and Remote Sensing Best Reviewers in 1999 and was a Guest Editor of a Special Issue of the IEEE TRANSACTIONS ON GEOSCIENCE AND REMOTE SENSING on the subject of the analysis of multitemporal remote sensing images (November 2003). He was the General Chair and Co-chair of the First and Second, respectively, IEEE International Workshop on the Analysis of Multitemporal Remote-Sensing Images. Since 2003, he has been the Chair of the SPIE Conference on Image and Signal Processing for Remote Sensing. He is an Associate Editor of the IEEE GEOSCIENCE AND REMOTE SENSING LETTERS. $\mathrm{He}$ is a member of the International Association for Pattern Recognition (IAPR) and of the Italian Association for Remote Sensing (AIT). 


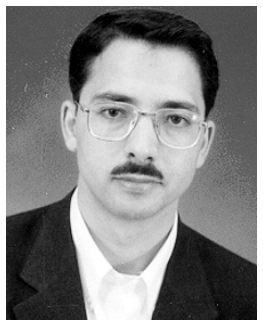

Farid Melgani (M'04) received the State Engineer degree in electronics from the University of Batna, Batna, Algeria, in 1994, the M.Sc. degree in electrical engineering from the University of Baghdad, Baghdad, Iraq, in 1999, and the Ph.D. degree in electronic and computer engineering from the University of Genoa, Genoa, Italy, in 2003.

From 1999 to 2002, he cooperated with the Signal Processing and Telecommunications Group, Department of Biophysical and Electronic Engineering, University of Genoa. He is currently an Assistant Professor of telecommunications at the University of Trento, Trento, Italy, where he teaches pattern recognition, radar remote sensing systems, and digital transmission. His research interests are in the area of processing and pattern recognition techniques applied to remote sensing images (classification, multitemporal analysis, and data fusion). He is coauthor of more than 30 scientific publications and is a referee for several international journals.

Dr. Melgani has served on the Scientific Committee of the SPIE International Conferences on Signal and Image Processing for Remote Sensing since 2000. 